\title{
Antioxidative and photocytotoxic effects of standardized Clinacanthus nutans and Strobilanthes crispus extracts toward HepG2 liver cells
}

\begin{abstract}
Introduction: The methanolic extracts of Clinacanthus nutans (CME) and Strobilanthes crispus (SME) are used in Malaysia as a complementary and alternative medicine for cancer.

Objective: The present study aimed to determine the antioxidative and photocytotoxic effects of CME and SME toward liver cancer cells.

Materials and Methods: Cell-based (2',7'-dichlorodihydrofluorescein diacetate) and chemical-based (2,2-diphenyl-1-picrylhydrazyl [DPPH]) experiments were utilized to determine the antioxidative properties of both herbal extracts. CME and SME were also tested for their photocytotoxic potentials after photodynamic therapy (PDT). Phytochemical analysis was performed to identify the phytocompounds present in the extracts.

Results: Both the extracts demonstrated dose-dependent DPPH radical scavenging activities, while SME was found to be a stronger reactive oxygen species scavenger than CME at all concentrations tested on liver cells. Interestingly, on PDT, HepG2 cells treated with SME and $\mathrm{CME}$ at nontoxic doses showed a decrease in cellular viability charting half-maximal inhibitory concentration of $13.45 \mu \mathrm{g} / \mathrm{mL}$ and $81.03 \mu \mathrm{g} / \mathrm{mL}$, respectively. Total phenolic content of SME $(36.27 \pm 1.31 \mathrm{mg} \mathrm{GAE} / \mathrm{g}$ extract) was slightly higher than CME (31.76 \pm $0.10 \mathrm{mg} \mathrm{GAE} / \mathrm{g}$ extract). On the contrary, the total flavonoid content of CME $(11.32 \pm 0.28$ $\mathrm{mg} \mathrm{QE} / \mathrm{g}$ extract) was approximately seven times more than SME $(1.69 \pm 0.03 \mathrm{mg} \mathrm{QE} / \mathrm{g}$ extract). Phenolic acids, flavonoids, and pheophorbide-a were identified in both extracts. In view of this, these phytocompounds present in CME and SME could lead to the observed beneficial effects.
\end{abstract}

Conclusion: CME and SME, especially the latter, are strong antioxidants with photosensitizing potentials that should be further investigated.

Keyword: Clinacanthus nutans; HepG2; Photocytotoxic; Photodynamic therapy; Reactive oxygen species; Strobilanthes crispus 